\title{
BMJ Open Randomised trials of proton pump inhibitors for gastro-oesophageal reflux disease in patients with asthma: an updated systematic review and meta- analysis
}

\author{
Zhoude Zheng, ${ }^{1}$ Yunyun Luo, ${ }^{2}$ Jia Li, ${ }^{1}$ Jinming Gao (i) ${ }^{1}$
}

To cite: Zheng Z, Luo Y, Li J, et al. Randomised trials of proton pump inhibitors for gastro-oesophageal reflux disease in patients with asthma: an updated systematic review and meta-analysis. BMJ Open 2021;11:e043860. doi:10.1136/ bmjopen-2020-043860

- Prepublication history and additional supplemental material for this paper are available online. To view these files, please visit the journal online. To view these files, please visit the journal online (http://dx.doi. org/10.1136/bmjopen-2020043860).

$\mathrm{ZZ}$ and YL contributed equally.

Received 15 August 2020 Accepted 09 June 2021

Check for updates

(C) Author(s) (or their employer(s)) 2021. Re-use permitted under CC BY-NC. No commercial re-use. See rights and permissions. Published by BMJ.

${ }^{1}$ Department of Pulmonary and Critical Care Medicine, Peking Union Medical College Hospital, Beijing, China

2Department of Endocrinology, Peking Union Medical College Hospital, Beijing, China

Correspondence to

Dr Jinming Gao;

gjinming@yahoo.com

\section{ABSTRACT}

Objective Asthma often coexists with gastro-oesophageal reflux disease (GERD). The effect of proton pump inhibitors (PPIs) treatment on asthma concomitant with GERD was inconsistent. This study aimed to assess whether PPIs treatment improved morning peak expiratory flow (mPEF) in asthma patients with GERD.

Data sources PubMed, MEDLINE, EMBASE, Web of Science, Cochrane Library and ClinicalTrials.gov; hand searching for reference lists; contacted with authors if necessary.

Study selection All eligible trials were randomised clinical trials comparing PPIs with placebo in asthma patients accompanying with GERD.

Results Fourteen randomised clinical trials (2182 participants) were included. Overall, PPIs versus placebo did not affect mPEF in patients with asthma having GERD (weighted mean difference $8.68 \mathrm{~L} / \mathrm{min}, 95 \% \mathrm{Cl}-2.02$ to 19.37, $p=0.11$ ). Trial sequential analysis (TSA) further confirmed this finding (TSA adjusted $95 \% \mathrm{Cl}-1.03$ to 22.25). Subgroups analyses based on the percentage of patients with symptomatic GERD $\geq 95 \%$, treatment duration $>12$ weeks also found no statistically significant benefit on mPEF. Similarly, analyses of secondary outcomes (evening PEF, forced expiratory volume in $1 \mathrm{~s}$, asthma symptoms score, asthma quality of life score and episodes of asthma exacerbation) did not show significant difference between PPIs and placebo.

Conclusion In this meta-analysis, PPIs therapy did not show a statistically significant improvement on $\mathrm{mPEF}$ in asthma patients having GERD, neither in subgroup with symptomatic GERD nor in subgroup with treatment duration $>12$ weeks. This analysis does not support a recommendation for PPIs therapy as empirical treatment in asthma patients with GERD.

PROSPERO registration number CRD42020177330.

\section{INTRODUCTION}

Asthma is a common chronic respiratory disease affecting approximately 300 million people worldwide. ${ }^{1} 2$ Gastro-oesophageal reflux disease (GERD) develops when the reflux of gastric contents causes irritating
Strengths and limitations of this study

- This systematic review strictly followed the methodology recommendations of the Cochrane Handbook, together with a comprehensive literature search.

- This study was carried out in accordance with the Preferred Reporting Items for Systematic Reviews and Meta-Analyses statement and its study protocol was registered on PROSPERO.

- We conducted trial sequential analysis in our outcomes as well as their subgroups analysis.

- The current study performed a cumulative metaanalysis in all the data.

- Some of the unreported raw data were still unavailable after making extensive efforts to obtain.

symptoms or complications, or both. ${ }^{3}$ GERD was considered as a trigger factor for asthma. Symptoms and/or diagnosis of GERD presented in $30 \%-90 \%$ of patients with asthma. ${ }^{4-6}$ Association between asthma and GERD has been extensively described elsewhere.$^{78}$ However, evidence of the causal link between asthma and GERD remains controversial. Some studies have shown that asthma may facilitate the development of GERD by the various mechanisms. ${ }^{78}$

Proton pump inhibitors (PPIs) were regarded as the cornerstone of antacid therapy and have been proved effective in empiric treatment of GERD. ${ }^{9}$ Given that GERD may be a risk factor for asthma, many randomised controlled trials (RCTs) were performed to identify the efficacy of different types of PPIs in the asthma patients with GERD. ${ }^{10-23}$ However, the efficacy of PPIs for the patients with asthma accompanying with GERD has been inconsistent. Previous metaanalyses have pooled the results of PPIs on asthma outcomes in children and adults, but all of them included a small sample size. ${ }^{24-26}$ 
The most recent systematic review examined the efficacy of PPIs treatment for the adults with asthma. However, the review only involved morning peak expiratory flow (mPEF) in subgroup of asthmatic patients diagnosed with GERD, and failed to identify the clinical characteristics of this subgroup population. ${ }^{27}$

Thus, we did a systematic review and meta-analyses to compare the effects PPIs versus placebo on asthma outcomes in the patients with GERD. Trial sequential analysis (TSA) was performed to quantify the meta-analysis monitoring boundaries and required information size (RIS) for primary outcome. Asthma outcomes included mPEF (primary outcome), evening peak expiratory flow (ePEF), forced expiratory volume in $1 \mathrm{~s}\left(\mathrm{FEV}_{1}\right)$, asthma symptoms score, asthma quality of life, episodes of asthma exacerbation.

\section{METHOD AND ANALYSIS}

The systematic review and meta-analyses were carried out in accordance with the Preferred Reporting Items for Systematic Reviews and Meta-Analyses statement. The protocol has been registered with International Prospective Register of Systematic Reviews (PROSPERO).

\section{Eligibility criteria}

Types of study

All randomised clinical trials of PPIs in the patients with asthma and GERD were included. The eligible randomised trials were required to report at least one clinical asthma outcome of interest.

\section{Types of participants}

Participants with asthma and GERD were eligible for inclusion. There were no restrictions regarding age, gender and ethnicity. Asthma was diagnosed according to doctor's diagnosis, reported ongoing asthma-related symptoms, evidence of objective measures of lung function. GERD diagnosis based on doctors' diagnosis, reported clinical symptoms of GERD and objective documentation.

\section{Types of intervention and control}

Trials comparing beneficial and harmful effects of PPIs with those of placebo were eligible. This review was restricted to studies with treatment duration of at least 4 weeks. ${ }^{27}$ No restrictions were imposed on drug dosage and types of PPIs which contained omeprazole, lansoprazole, pantoprazole, esomeprazole and rabeprazole. We excluded the trials that focused on the intervention with combination of PPIs and other antacids or gastrointestinal motility regulators.

\section{Outcome measures}

This review evaluated the following outcomes: mPEF, ePEF and $\mathrm{FEV}_{1}$, which were commonly used as evidence of variable expiratory airflow obstruction. Other outcomes included asthma symptoms score (validated questionnaires of all types), asthma quality of life (validated instruments of all types), episodes of asthma exacerbation and adverse events.

\section{Information sources and search}

A systematic search for evidence on the efficacy of PPIs on patients with asthma was performed through electronic databases, citation search based on reference lists and hand searching of main relevant journals. We did a search in PubMed, EMBASE, Web of Science, Cochrane Library and ClinicalTrials.gov dating from inception to 18 March 2020. No restrictions were imposed on language, publication date, publication type or publication status. The search terms and search strategies for all databases were described in online supplement 1 .

\section{Study selection}

Two reviewers (ZZ and YL) independently screened titles and abstracts according to the eligibility criteria in an unblinded, standardised manner. Reviews, letters, editorials, case studies, non-human studies, study protocols, non-English-language abstract were excluded during this process. The assessments of eligible full-text articles were carried out independently by two reviewers (ZZ and YL). Disagreements between reviewers were resolved by consensus or referred to a third reviewer (JG) for resolution.

\section{Data extraction}

Two independent reviewers (ZZ and YL) extracted data from each eligible study by using a predesigned extraction form. Discrepancies were resolved by consensus or by involvement of a third author (JG). Items of characteristics of included studies were described in online supplement 1 . We contacted the corresponding authors for outcomes data if required.

\section{Risk of bias in individual studies}

Two independent reviewers (ZZ and YL) evaluated risk of bias according to version 5.1.0 of Cochrane Handbook for Systematic Review of Interventions. An agreement was reached by discussion or by consultation with a third review author (JG). The domains of evaluation for all the outcomes were selection bias, performance bias, detection bias, attrition bias, reporting bias and other bias. Each potential source of bias was considered as either 'high risk', 'low risk' or 'unclear risk'.

\section{Statistical analysis}

The weighted mean difference / standardised mean difference (SMD) and 95\% CIs were calculated for continuous outcomes. The relative risk with $95 \%$ CIs was calculated for dichotomous outcomes. Predefined subgroup analysis was undertaken in accordance with patients aged 18 years and older or patients younger than 18 years, the percentage of subjects with symptomatic GERD $\geq 95 \%$, treatment duration ( $\leq 12$ weeks vs $>12$ weeks) and types of PPIs (omeprazole, pantoprazole, lansoprazole, esomeprazole). Given the 
anticipated variability among patient characteristic and study design, a random effects model with 95\% CIs was used in the forest plots (RevMan V.5.3). Statistical heterogeneity was quantified using $\mathrm{I}^{2}$ statistic, with $\mathrm{I}^{2}$ cut-off value of $25 \%, 50 \%$ and $75 \%$ to quantify low, moderate and high thresholds, respectively. We adopted cumulative meta-analysis in all the data and conducted sensitivity analysis and Egger's test to identify data stability and publication bias, respectively (StataSE V.12.0). TSA (V.0.9.5.10 Beta) was performed in $\mathrm{mPEF}$ and ePEF to quantify meta-analysis monitoring boundaries and RIS using parameters of mean difference of $\mathrm{mPEF}=20 \mathrm{~L} / \mathrm{min}$, estimate variance from the meta-analysis of PEF data, $\alpha$ at 0.05 , power of $80 \%$, and $\mathrm{I}^{2}$ value of $0 \%$.

\section{Patient and public involvement}

There was no patient or public involvement in this study.

\section{RESULTS}

\section{Study selection and characteristics}

The search strategy yielded 2005 abstracts, of which 49 abstracts were retrieved and under full-articles assessment for eligible articles. All studies conducted lasted for more than 4 weeks. Of these trials, 14 RCTs were included, 6 of which were cross-over studies, ${ }^{10-12141520}$ and 8 were of a parallel design. ${ }^{13}$ 16-19 21-23 The flow diagram for study inclusion is described in figure 1 . Table 1 and online supplemental table 1 summarise the characteristics of the included studies (2182 participants) and the characteristics of the subjects, respectively. Of the 14 eligible trials, 12 included subjects aged $\geq 18$ years, while only 2 aimed at patients aged $<18$ years (ranged from 6 to 17 years old). ${ }^{1723}$ Mild to severe asthmatics were included. The severity of GERD was reported inconsistently among the trials. Symptoms of heartburn, regurgitation and dysphagia were the common presentations of GERD reported in most studies. The percentage of the subjects

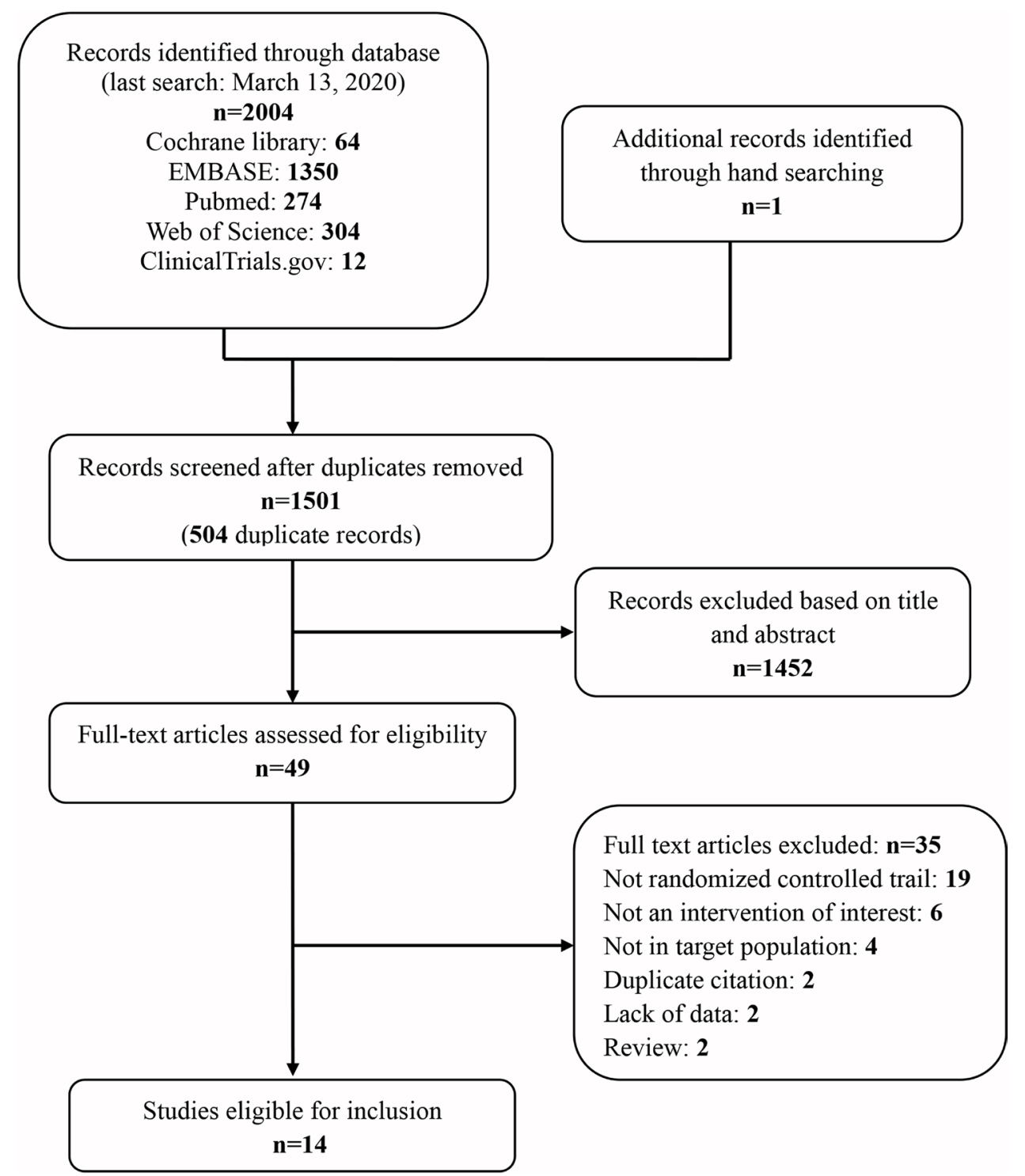

Figure 1 Flow diagram of identification of eligible studies for inclusion. 


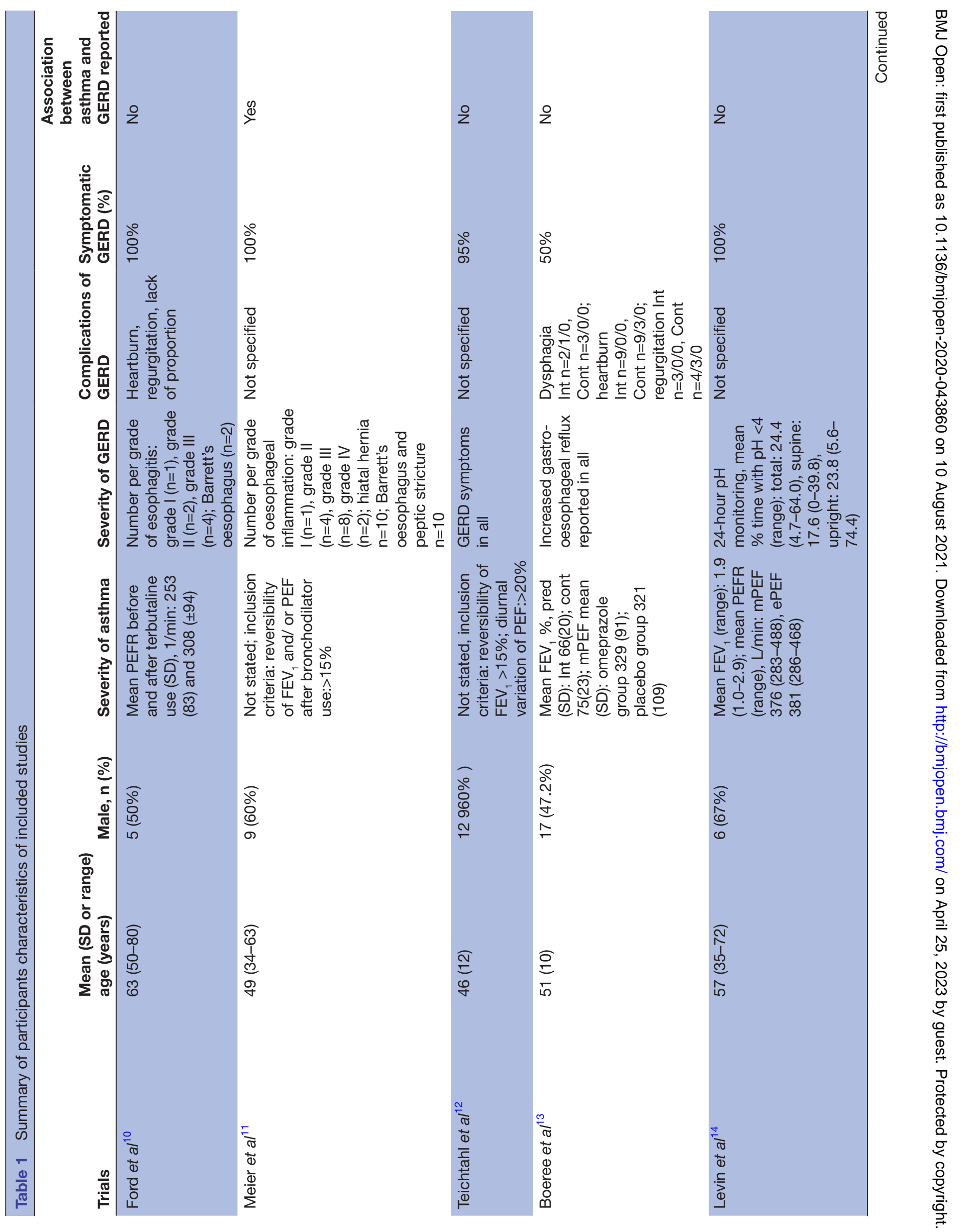




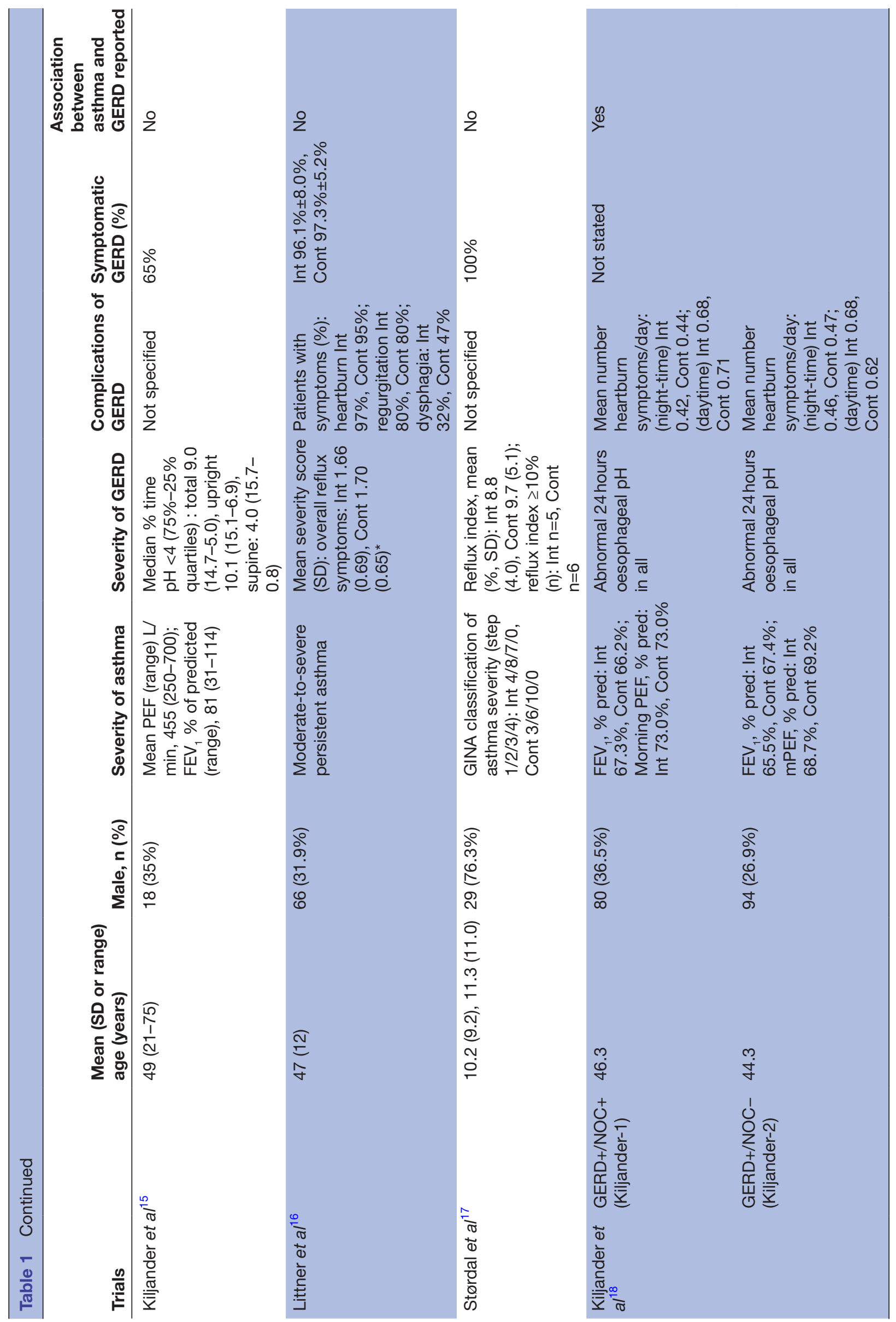




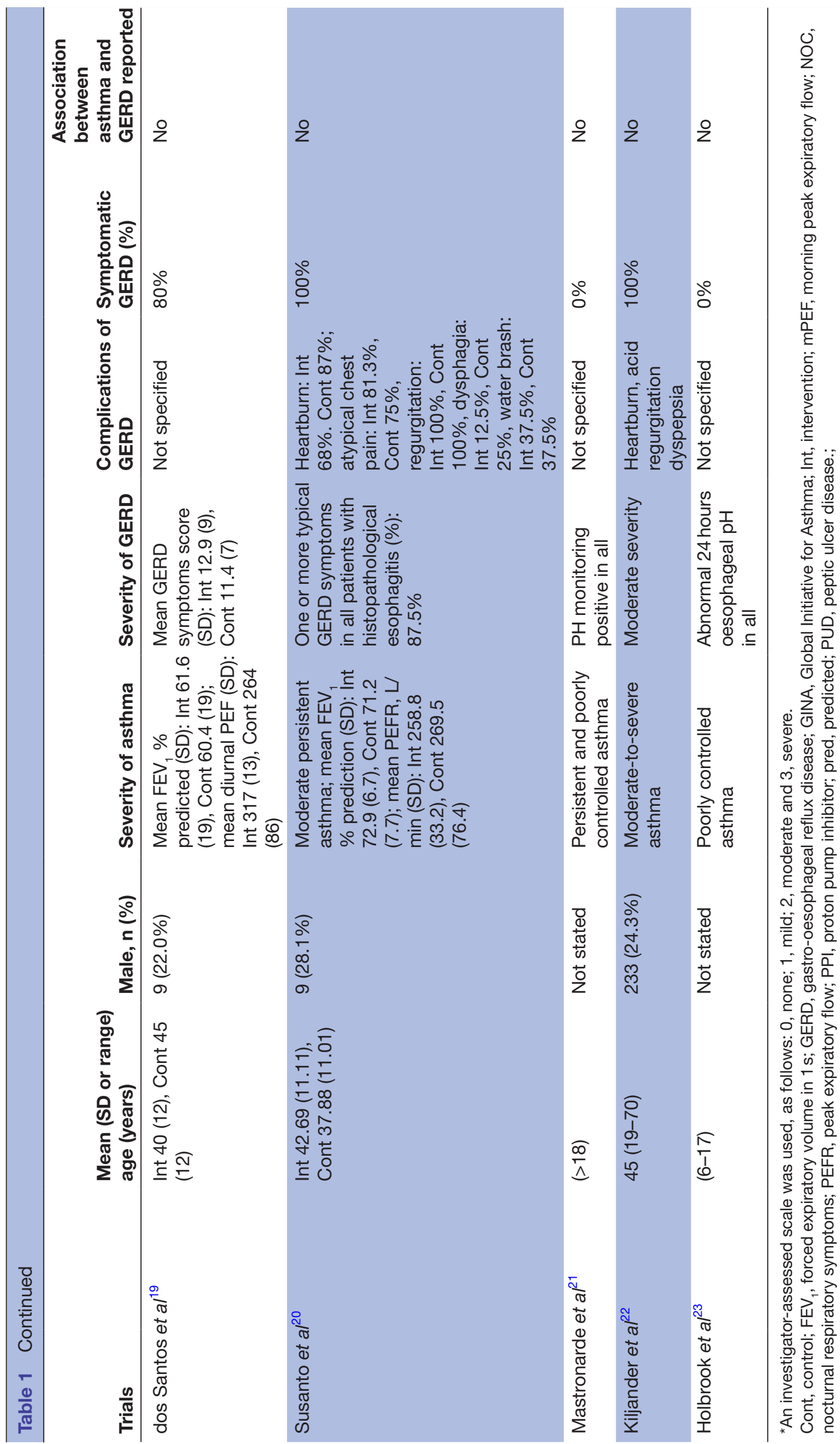

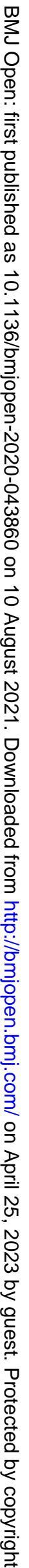




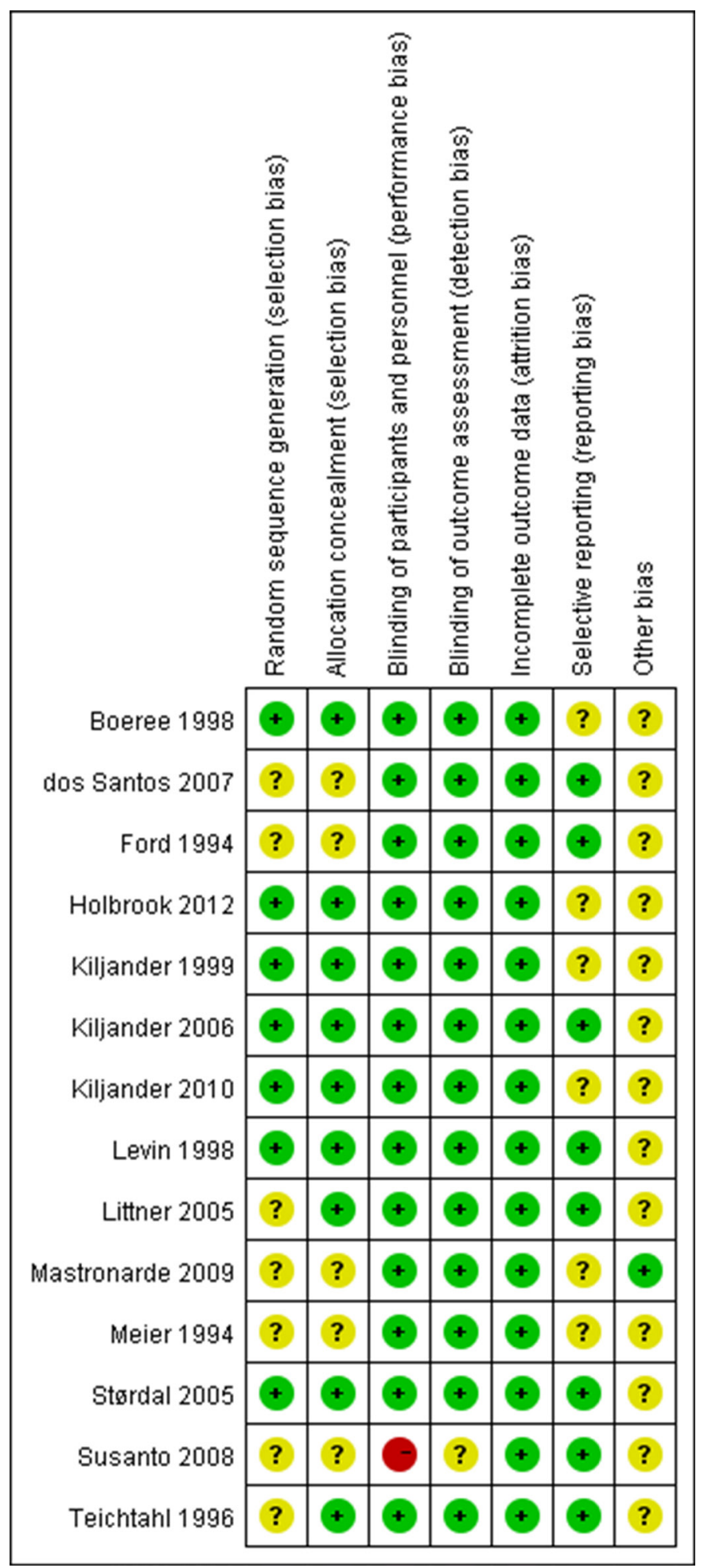

Figure 2 Risk of bias summary displaying review authors' judgements about each risk of bias item for each included study.

with symptomatic GERD was greater than $95 \%$ in eight studies, of which six studies reported $100 \%$. $^{101114172022}$

\section{Risk of bias within studies}

Each study was assessed in accordance with the Cochrane risk of bias tool (figure 2). ${ }^{28}$ Double-blinding method was adopted in all studies except one trial which used a single-blinding fashion. ${ }^{20}$ Three trials were supported by pharmaceutical companies. ${ }^{16} 1822$

\section{Outcomes}

Fourteen included studies investigated PPIs therapy on patients with asthma and GERD (2182 patients). Asthma outcomes were reported inconsistently among studies, leading to limitation of meta-analysis (table 2). All studies reported one or more outcomes of lung function.

\section{Primary outcome}

Morning PEF

Only one of the studies with data available found a significant improvement on mPEF. ${ }^{19}$ Eight studies containing nine groups were included in meta-analysis (1886 subjects). Among the nine groups, eight showed improvement in asthma symptoms, ${ }^{10} 121316$ 18-20 22 but only one group did not cross the neutral (zero) line. ${ }^{19}$ The overall analysis found no statistically significant benefit on $\mathrm{mPEF}$ with PPIs treatment $(8.68 \mathrm{~L} / \mathrm{min}, 95 \% \mathrm{CI}-2.02$ to 19.37 , $\mathrm{p}=0.11)$. Heterogeneity was absent $\left(\mathrm{I}^{2}=0 \% ; \mathrm{p}=0.73\right)$ (figure 3A). TSA showed a heterogeneity adjusted RIS of 1240 patients without the cumulative $\mathrm{Z}$ curve crossing boundaries for benefit or harm (TSA adjusted 95\% CI -1.03, 22.25), suggesting that PPIs may not show benefit on mPEF of the patients with asthma and GERD (figure 4A). No publication bias reported in $\mathrm{mPEF}$, and the sensitivity analysis confirmed the robustness of these findings (online supplemental figure 1).

A subgroup was performed according to the percentage of subjects with symptomatic GERD $\geq 95 \%$ (1253 participants). Of eight eligible studies, five reported available data for meta-analysis. ${ }^{1012162022}$ No statistically significant effect was found for $\mathrm{mPEF}$ in this subgroup $(7.07 \mathrm{~L} / \mathrm{min}$, $95 \%$ CI -6.56 to $20.69, \mathrm{p}=0.31$ ) (figure 3B). TSA showed that only $1158(79 \%)$ of the heterogeneity adjusted RIS of 1470 patients were calculated. However, the cumulative $\mathrm{Z}$ curve crossed the boundaries for futility (TSA adjusted $95 \%$ CI -5.94 to 25.58 ) (figure $4 \mathrm{~B}$ ).

Next, we conducted subgroups analysis based on duration of PPIs treatment (duration $\leq 12$ weeks with a population of 164 vs $>12$ weeks with 1722 participants). No statistically significant benefit was demonstrated in both subgroups (duration $\leq 12$ weeks: $23.06 \mathrm{~L} / \mathrm{min}, 95 \%$ CI -3.40 to $49.51, \mathrm{p}=0.09, \mathrm{p}=0.43$; duration $>12$ weeks: $5.87 \mathrm{~L} / \mathrm{min}, 95 \% \mathrm{CI}-5.83$ to $17.56, \mathrm{p}=0.33$ ) (figure $3 \mathrm{C}$ ). Then we conducted TSA in the subgroup with duration $>12$ weeks. TSA did not alter the efficacy on mPEF with a PPIs treatment duration $>12$ weeks (TSA adjusted 95\% CI -4.99 to 20.50 ) (figure 4C).

Also, three subgroups meta-analyses based on types of PPIs did not show statistically significant treatment benefit (omeprazole: 88 subjects, $4.65 \mathrm{~L} / \mathrm{min}, 95 \% \mathrm{CI}-35.43$ to 44.72, p=0.82; lansoprazole: 251 subjects, $29.18 \mathrm{~L} / \mathrm{min}$, 95\% CI -23.21 to $81.56, \mathrm{p}=0.27$; esomeprazole: 1547 subjects, $5.91 \mathrm{~L} / \mathrm{min}, 95 \% \mathrm{CI}-7.02$ to $18.84, \mathrm{p}=0.37$ ) on mPEF (figure 3D). 
Table 2 Summary of results of proton pump inhibitors treatment on asthma outcomes

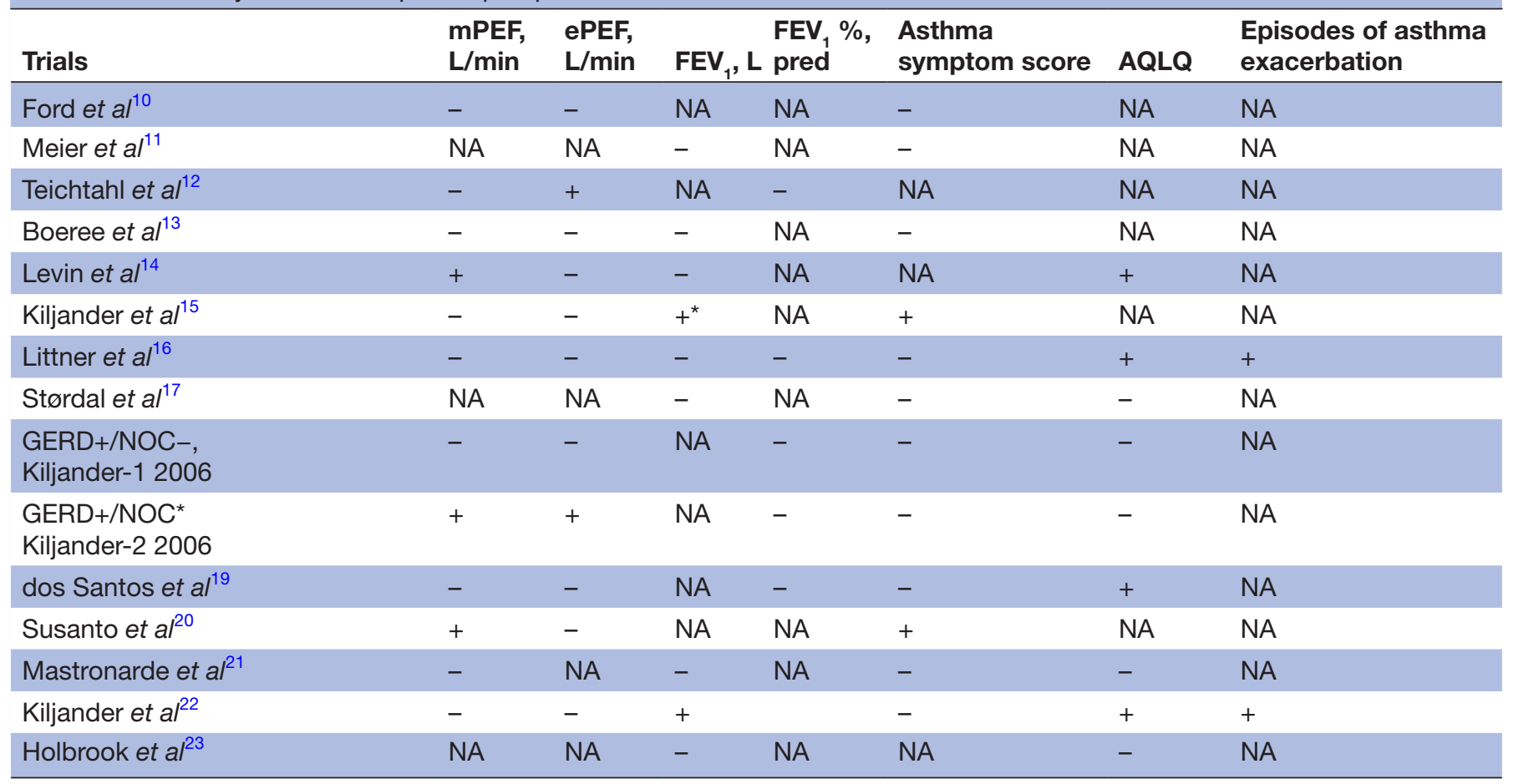

+, significant therapy effect; -, not significant therapy effect.

*Decline during omeprazole use.

AQLQ, Asthma Quality of Life Questionnaire; ePEF, evening peak expiratory flow; FEV , forced expiratory volume in $1 \mathrm{~s}$; mPEF, morning peak expiratory flow; NA, not available; pred, predicted.

We carried out a cumulative meta-analysis of the effect of PPIs on the MPEF and its subgroups analysis based on the data of publication. However, the effect of PPIs remained unchanged (online supplemental figure 2).

\section{Secondary outcomes \\ Evening PEF}

Ten trials reported ePEF of the subjects with asthma and GERD, of which two trials demonstrated statistically significant improvement on ePEF. ${ }^{12} 18$ Of these 10 trials, 6 studies provided information and were included in the meta-analyses (901 participants). ${ }^{10} 1216{ }^{18-20}$ Meta-analysis did not show statistically significant effect on ePEF ( $5.58 \mathrm{~L} / \mathrm{min} ; 95 \% \mathrm{CI}-8.19$ to $19.36, \mathrm{p}=0.43$ ) (figure $5 \mathrm{~A}$ ). TSA showed that the cumulative $\mathrm{Z}$ curve crossed boundaries for futility, suggesting no statistically significant improvement on ePEF with PPIs therapy (TSA adjusted $95 \% \mathrm{CI}-6.87$ to 25.35$)$. No publication bias reported in $\mathrm{ePEF}$, and the sensitivity analysis showed solid results (online supplemental figure 3A).

No statistically significant benefit was showed on ePEF by subgroups analyses of the studies in accordance with the percentage of subjects with symptomatic GERD $\geq 95 \%$, length of PPIs treatment and types of PPIs (online supplemental figure $3 \mathrm{~B}$ ).

\section{Forced expiratory volume in $1 \mathrm{~s}$}

Three studies with a population of 640 provided information of $\mathrm{FEV}_{1} \%$ predicted, ${ }^{12} 1819$ and only two with
237 participants provided available data of $\mathrm{FEV}_{1}(\mathrm{~L}),{ }^{13} 16$ which were included in analyses, respectively. At the analysis of $\mathrm{FEV}_{1} \%$ predicted, no therapy effect was found on the patients with PPIs use $(-1.25 \%, 95 \%$ CI -4.9 to $3.00, \mathrm{p}=0.56$ ) (figure 5B1). Heterogeneity was substantial $\left(\mathrm{I}^{2}=61 \% ; \mathrm{p}=0.05\right)$. The analysis of the two studies may not demonstrated a benefit on the $\mathrm{FEV}_{1}(\mathrm{~L})$ in the patients with PPIs therapy $(-0.09 \mathrm{~L}, 95 \% \mathrm{CI}-0.28$ to 0.10 , $\mathrm{p}=0.36$ ) (figure 5B2). No publication reported in $\mathrm{FEV}_{1} \%$ predicted, the sensitivity analysis showed robust results (online supplemental figure 4).

\section{Asthma symptoms score}

Six studies reported information of asthma symptoms score and were included in meta-analysis (371 participants). ${ }^{101316171920}$ Five of six trials included the patients aged older than 18 years (335 participants). The subgroup of adults showed no statistically significant effect on asthma symptoms score with PPIs treatment (SMD $-0.30,95 \% \mathrm{CI}-0.61$ to $0.01, \mathrm{p}=0.06$, heterogeneity $\mathrm{I}^{2}=32 \%, \mathrm{p}=0.21$ ). However, the analysis found a small statistically significant improvement on asthma symptoms score (SMD $-0.26,95 \% \mathrm{CI}-0.52$ to $-0.01, \mathrm{p}=0.04$ ), when we pooled the studies in adults and those in children. Heterogeneity was low $\left(\mathrm{I}^{2}=19 \%, \mathrm{p}=0.29\right)$ (figure $5 \mathrm{C}$ ). No publication reported in asthma symptoms score, and the sensitivity analysis showed that the results were robust (online supplemental figure 5). 
A

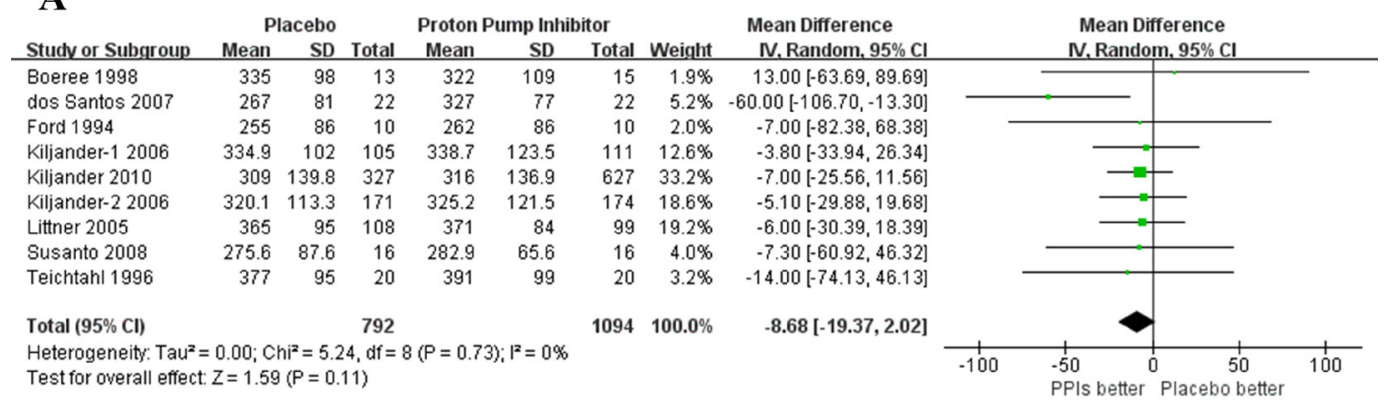

\section{B}

\begin{tabular}{|c|c|c|c|c|c|c|c|c|c|c|c|c|}
\hline \multirow[b]{2}{*}{ Study or Subgroup } & \multicolumn{3}{|c|}{ Placebo } & \multicolumn{3}{|c|}{ Proton Pump Inhibitor } & \multirow[b]{2}{*}{ Weight } & \multirow{2}{*}{$\begin{array}{l}\text { Mean Difference } \\
\text { IV. Random, 95\% Cl }\end{array}$} & \multirow{2}{*}{\multicolumn{4}{|c|}{$\begin{array}{c}\text { Mean Difference } \\
\text { IV.Random, } 95 \% \mathrm{Cl}\end{array}$}} \\
\hline & Mean & SD & Total & Mean & SD & Total & & & & & & \\
\hline Ford 1994 & 255 & 86 & 10 & 262 & 86 & 10 & $3.3 \%$ & $-7.00[-82.38,68.38]$ & & & & \\
\hline Kiljander 2010 & 309 & 139.8 & 327 & 316 & 136.9 & 627 & $53.9 \%$ & $-7.00[-25.56,11.56]$ & & & & \\
\hline Littner 2005 & 365 & 95 & 108 & 371 & 84 & 99 & $31.2 \%$ & $-6.00[-30.39,18.39]$ & & & & \\
\hline Susanto 2008 & 275.6 & 87.6 & 16 & 282.9 & 65.6 & 16 & $6.5 \%$ & $-7.30[-60.92,46.32]$ & & & & \\
\hline Teichtahl 1996 & 377 & 95 & 20 & 391 & 99 & 20 & $5.1 \%$ & $-14.00[-74.13,46.13]$ & & & & \\
\hline Total $(95 \%$ Cl) & & & 481 & & & 772 & $100.0 \%$ & $-7.07[-20.69,6.56]$ & & & & \\
\hline $\begin{array}{l}\text { Heterogeneity: Tauz } \\
\text { Test for overall effect }\end{array}$ & $\begin{array}{l}0.00 ; \mathrm{Cr} \\
z=1.02\end{array}$ & $\begin{array}{l}h^{2}=0.0 \\
(P=0 .\end{array}$ & $\begin{array}{l}\text { 6, } \mathrm{df}=4 \\
\text { 31) }\end{array}$ & $(P=1.0$ & $1^{2}=0 \%$ & & & & -100 & $\begin{array}{l}-50 \\
\text { PPIs better }\end{array}$ & ${ }^{0} \quad 50$ & $\begin{array}{ll}50 & 100 \\
\text { better } & \end{array}$ \\
\hline
\end{tabular}

C

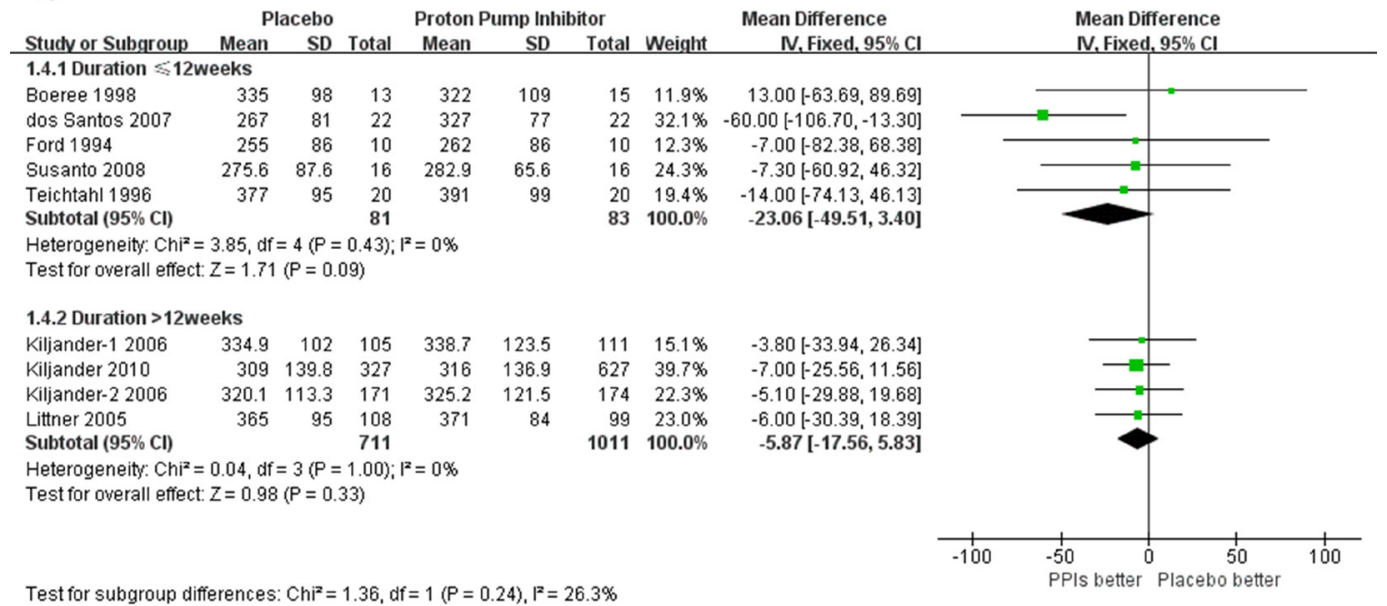

D

\begin{tabular}{|c|c|c|c|c|c|c|c|c|c|c|c|c|}
\hline \multirow[b]{2}{*}{ Study or Subgroup } & \multicolumn{3}{|c|}{ Placebo } & \multicolumn{3}{|c|}{ Proton Pump Inhibitor } & & \multirow{2}{*}{$\begin{array}{l}\text { Mean Difference } \\
\text { IV. Random, } 95 \% \mathrm{Cl}\end{array}$} & \multirow{2}{*}{\multicolumn{4}{|c|}{$\begin{array}{c}\text { Mean Difference } \\
\text { IV, Random, } 95 \% \mathrm{Cl}\end{array}$}} \\
\hline & Mean & SD & Total & Mean & SD & Total & Weight & & & & & \\
\hline \multicolumn{13}{|c|}{ 1.3.1 Omeprazole group } \\
\hline Boeree 1998 & 335 & 98 & 13 & 322 & 109 & 15 & $27.3 \%$ & $13.00[-63.69,89.69]$ & & & & \\
\hline Ford 1994 & 255 & 86 & 10 & 262 & 86 & 10 & $28.3 \%$ & $-7.00[-82.38,68.38]$ & & & & \\
\hline Teichtahl 1996 & 377 & 95 & 20 & 391 & 99 & 20 & $44.4 \%$ & $-14.00[-74.13,46.13]$ & & & & \\
\hline Subtotal (95\% Cl) & & & 43 & & & 45 & $100.0 \%$ & $-4.65[-44.72,35.43]$ & & & & \\
\hline \multicolumn{13}{|c|}{$\begin{array}{l}\text { Heterageneity: } \mathrm{Tau}^{2}=0.00 ; \mathrm{Chi}^{2}=0.30, \mathrm{df}=2(P=0.86) ;\left.\right|^{2}=0 \% \\
\text { Test for overall effect: } Z=0.23(P=0.82)\end{array}$} \\
\hline \multicolumn{13}{|c|}{ 1.3.2 Lansoprazole group } \\
\hline dos Santos 2007 & 267 & 81 & 22 & 327 & 77 & 22 & $42.9 \%$ & $-60.00[-106.70,-13.30]$ & & & & \\
\hline Littner 2005 & 365 & 95 & 108 & 371 & 84 & 99 & $57.1 \%$ & $-6.00[-30.39,18.39]$ & & & & \\
\hline Subtotal $(95 \% \mathrm{Cl})$ & & & 130 & & & 121 & $100.0 \%$ & $-29.18[-81.56,23.21]$ & & & & \\
\hline \multicolumn{13}{|c|}{$\begin{array}{l}\text { Heterogeneity: } \text { Tau }^{2}=1096.72 ; \mathrm{Chi}^{2}=4.04, \mathrm{df}=1(\mathrm{P}=0.04) ; \mathrm{F}^{2}=75 \% \\
\text { Test for overall effect: } Z=1.09(\mathrm{P}=0.27)\end{array}$} \\
\hline \multicolumn{13}{|c|}{ 1.3.3 Esomeprazole group } \\
\hline Kiljander-1 2006 & 334.9 & 102 & 105 & 338.7 & 123.5 & 111 & $18.4 \%$ & $-3.80[-33.94,26.34]$ & & & & \\
\hline Kiljander 2010 & 309 & 139.8 & 327 & 316 & 136.9 & 627 & $48.6 \%$ & $-7.00[-25.56,11.56]$ & & $\longrightarrow$ 맘 & - & \\
\hline Kiljander-2 2006 & 320.1 & 113.3 & 171 & 325.2 & 121.5 & 174 & $27.2 \%$ & $-5.10[-29.88,19.68]$ & & & & \\
\hline Susanto 2008 & 275.6 & 87.6 & 16 & 282.9 & 65.6 & 16 & $5.8 \%$ & $-7.30[-60.92,46.32]$ & & & & \\
\hline Subtotal $(95 \% \mathrm{Cl})$ & & & 619 & & & 928 & $100.0 \%$ & $-5.91[-18.84,7.02]$ & & & & \\
\hline \multicolumn{13}{|c|}{$\begin{array}{l}\text { Heterogeneity: } \mathrm{Tau}^{2}=0.00 ; \mathrm{Chi}^{2}=0.04, \mathrm{df}=3(\mathrm{P}=1.00) ; \mathrm{I}^{2}=0 \% \\
\text { Test for overall effect: } Z=0.90(\mathrm{P}=0.37)\end{array}$} \\
\hline & & & & & & & & & -100 & -50 & 50 & 100 \\
\hline
\end{tabular}

Figure 3 (A) Forest plot for morning peak expiratory flow (MPEF). (B) Forest plot for mPEF in subgroup of the percentage of subjects with symptomatic gastro-oesophageal reflux disease $\geq 95 \%$. (C) Forest plot for mPEF in subgroups of treatment duration $\leq 12$ weeks and $>12$ weeks. (D) Forest plot for mPEF in subgroups of different types of proton pump inhibitors. PPIs, proton pump inhibitors. 

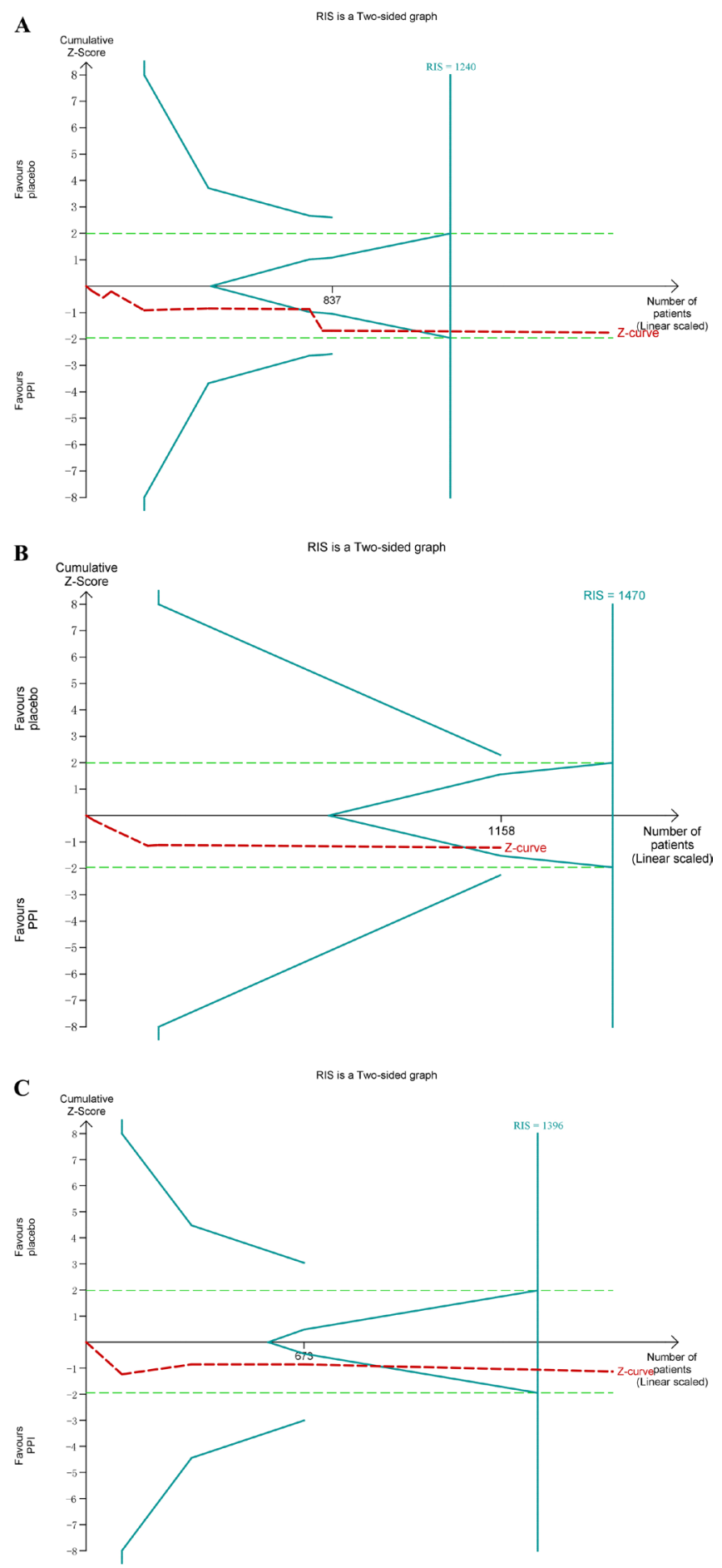

Figure 4 (A) Trial sequential analysis (TSA) of morning peak expiratory flow (MPEF). (B) TSA of MPEF in subgroup of the percentage of subjects with symptomatic gastrooesophageal reflux disease $\geq 95 \%$. (C) TSA of mPEF in subgroup of treatment duration $>12$ weeks. PPIs, proton pump inhibitors; RIS, required information size.

\section{Asthma quality of life}

Four eligible studies were included for meta-analysis (853 subjects). ${ }^{16181923}$ The result showed no overall effect on the asthma quality of life (SMD $0.01,95 \%$ CI -0.44 to $0.47, \mathrm{p}=0.96)$. Heterogeneity was substantial $\left(\mathrm{I}^{2}=89 \%\right.$, $\mathrm{p}<0.00001$ ) (figure 5D). No publication bias was reported in this outcome $(p=0.588)$, but sensitivity analysis showed the results were unstable (online supplemental figure 6). Therefore, the pooled result for asthma quality of life had limited meaning.

\section{Episodes of asthma exacerbation}

Only two studies including 1167 patients provided information of episodes of asthma exacerbation and showed an improvement in this variance. ${ }^{1622}$ However, no effect was showed in meta-analysis (relative risk $0.55,95 \%$ CI 0.21 to $1.43, \mathrm{p}=0.22)$. Heterogeneity was substantial $\left(\mathrm{I}^{2}=81 \%\right.$, $\mathrm{p}<0.02$ ) (figure $5 \mathrm{E}$ ).

Cumulative meta-analysis was performed in all the data of secondary outcomes. Similarly, except a minor improvement on asthma symptoms score, it was likely that no significant effect was found on ePEF, $\mathrm{FEV}_{1} \%$ predicted, asthma quality of life and episodes of asthma exacerbation with the application of PPIs (online supplemental figure 7 ).

\section{DISCUSSION}

For primary outcome mPEF, we assessed eight studies including nine independent comparisons (1886 participants) and found no statistically significant improvement with PPIs treatment in patients with asthma and GERD compared with placebo. Subgroups analyses according to duration $>12$ weeks and the percentage of subjects with symptomatic GERD $\geq 95 \%$, did not demonstrated statistically significant benefit with PPIs therapy. Also, no statistically significant improvement was observed on the secondary outcomes including ePEF, $\mathrm{FEV}_{1}$, asthma symptoms, quality of life and asthma exacerbation. These results were further confirmed by the application of TSA and cumulative meta-analysis.

To enlarge sample size, our analysis not only included trials with asthma subjects having GERD diagnosis for entry criterion, but also those reported GERD subjects in subgroups analyses. ${ }^{1820}$ To the best of our knowledge, this analysis included the largest number of participants to date describing the effect of PPIs treatment in patients with asthma accompanying with GERD. The previous meta-analysis aiming to examine the efficacy of PPIs in the adult patients with asthma, reported a subgroup analysis based on GERD diagnosis for entry criterion with seven trials (1004 patients).$^{27}$ In contrast to our study, a small statistically significant improvement was reported for $\mathrm{MPEF}$ in this subgroup, therefore, this analysis might overestimate the benefits on $\mathrm{mPEF}$ and exaggerate the effect of positive improvement, because of incomplete and inadequate population inclusion. However, in line with our results, this previous review did not show benefit on in patients with asthma with PPIs treatment on ePEF, $\mathrm{FEV}_{1}$, asthma symptoms score and asthma quality of life.

A study reported that the minimal patient perceivable improvement differences for PEF was $18.79 \mathrm{~L} / \mathrm{min}^{29}$ The minimal difference in PEF ranging from 15 to $20 \mathrm{~L} /$ 

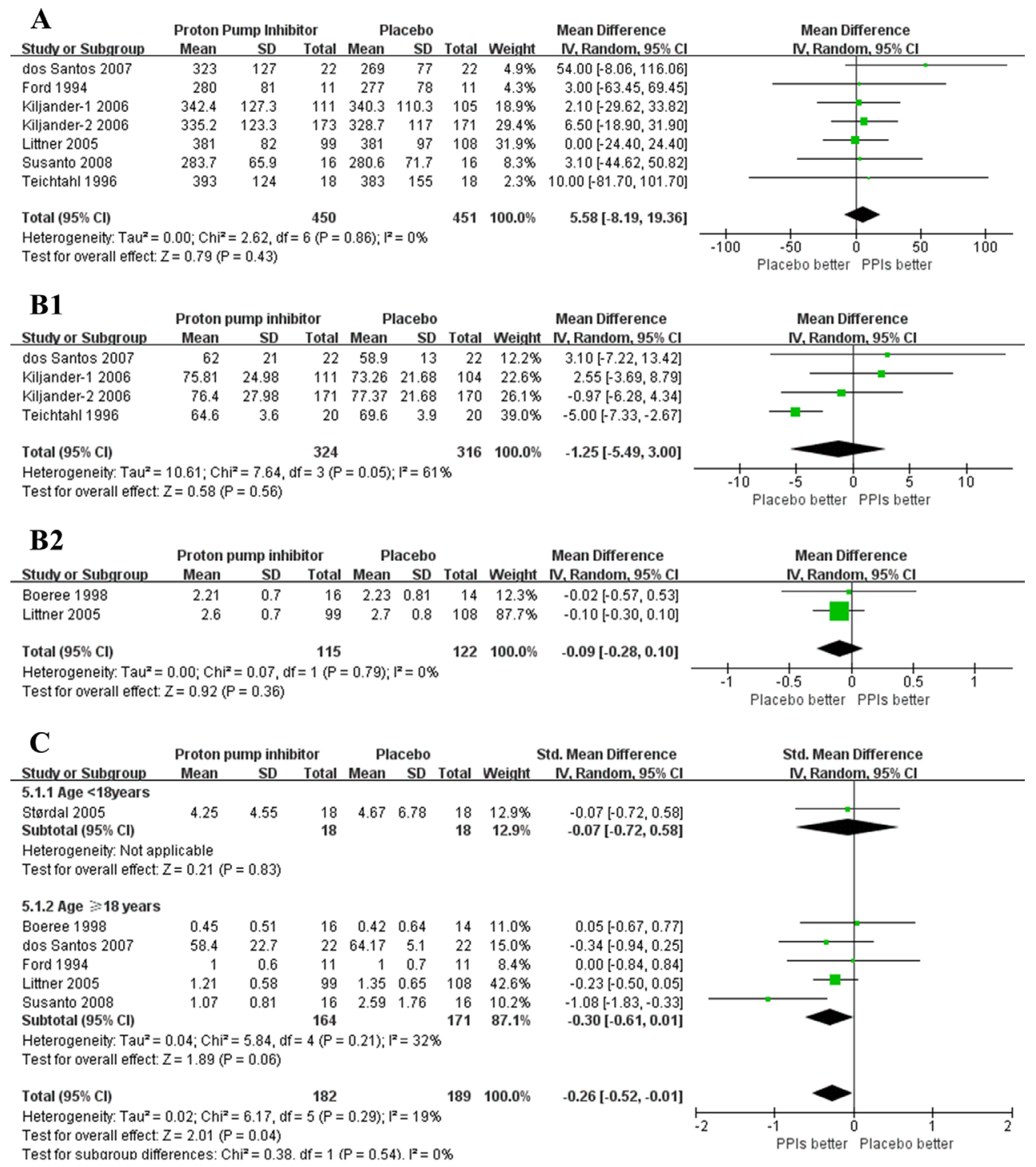

Test for subaroun differences: $\mathrm{Chi}^{2}=0.38 \mathrm{df}=1(\mathrm{P}=0.54) \cdot \mathrm{I}^{2}=0$.

Figure 5 (A) Forest plot for evening peak expiratory flow. (B1) Forest plot for FEV ${ }_{1} \%$ predicted. (B2) Forest plot for FEV 1 (L). (C) Forest plot for asthma symptoms score. (D) Forest plot for asthma quality of life score. (E) Forest plot for episodes of asthma exacerbation. $\mathrm{FEV}_{1}$, forced expiratory volume in $1 \mathrm{~s}$; PPIs, proton pump inhibitors. 
min were summarised in a review. ${ }^{30}$ Our analysis found that the pooled mean difference for $\mathrm{MPEF}$ and $\mathrm{ePEF}$ were 7.30 and $5.58 \mathrm{~L} / \mathrm{min}$, respectively, which were far smaller than the minimal effective line, probably showing a lack of evidence to believe the efficacy of PPIs. In alignment with our study, previous meta-analysis published by Cochrane Collaboration found no statistically significant improvement on mPEF and ePEF. ${ }^{25}$ Also, a recent large three-arms RCT was consistent with our study. ${ }^{22}$

Several trials have reported that PPIs played no role in asthma patients with asymptomatic GERD, whether in children or adults. ${ }^{21}{ }^{23}$ Similarly, in our subgroup metaanalysis, no statistically significant benefit appeared for mPEF in asthma patients with symptomatic GERD. This result was in keeping with a large trial including all asthma participants with symptomatic GERD. ${ }^{22}$ Our subgroup analysis for $\mathrm{mPEF}$ based on duration $>12$ weeks was conducted, suggesting that no improvement appeared with PPIs therapy. In agreement with our result, two large trials did not find improvement for MPEF with PPIs treatment for 24 or 26 weeks. ${ }^{162}$

Mechanistically, GERD may trigger asthma via directly damage to the respiratory tree leading to bronchoconstriction by micro-aspiration of gastric or duodenal (or both) contents. ${ }^{31}{ }^{32}$ Previous studies have reported that bile acids and pepsin were found graft failure in lung transplant patients, indicating that acid materials may not be the only one of many irritants in the aspirate during gastro-oesophageal reflux. ${ }^{33} 34$

PPIs treatment significantly improved asthma symptoms and lung function in patients with exercise-triggered asthma, with asthma and nocturnal respiratory symptoms, or taking LABAs. ${ }^{18}{ }^{35}$ It appeared that benefits of PPIs may be restricted to patients with certain types or status of asthma. Further studies are warranted to examine the pathophysiological mechanism to determine the causality between asthma and GERD. Notably, if the improvement for asthma conditions were delayed or required more time to present, then the overall effect may be underestimated. Thus, further RCTs should be conducted with a treatment period for more than 6 months. Previous RCTs combined omeprazole and domperidone therapy in patients with asthma and GERD, showing that combined therapy improved asthma symptoms and lung function with treatment period of 12 or 16 weeks. ${ }^{36} 37$ Therefore, the efficacy of combined therapy should be further explored. Furthermore, we hopefully expect the effect of genotype-tailored PPIs in patients with asthma and comorbid GERD. ${ }^{38}$

There are several limitations in the present study. First, we could not extract the data from all the 11 eligible trials reporting $\mathrm{mPEF}$, because of the unavailable reported form (mean difference only, ${ }^{14}$ medians and quartiles ${ }^{15}$ ) or unavailable data in subgroup. ${ }^{21}$ However, the overall sample size of these three trials was small and we do not think these studies would make a significant difference in our meta-analysis. Second, we could not perform a subgroup according to the severity of asthma or GERD as expected, because the severity reported inconsistently and we could not sort out the disease status of each trial. Third, only two RCTs in children were eligible in the present study, making it difficult to evaluate the effect for PPIs on all outcomes in children. ${ }^{17}{ }^{23}$ However, both trials reported no improvement for PPIs in all the asthma outcomes, which were in line with the overall effect in adults in our analysis.

\section{CONCLUSION}

Compared with placebo, PPIs therapy for asthma patients with GERD did not show statistically significant improvement in mPEF. This futility did not alter in asthma patients neither with symptomatic GERD nor with PPIs treatment for more than 12 weeks. This analysis does not support a recommendation for the empirical use of PPIs therapy in asthma patients having GERD.

Acknowledgements The authors would like to thank Dr Xiaoling Li for her suggestions on grammar and vocabularies of this manuscript. We would also like to thank Dr Xiaorui Lyu (Department of Endocrinology, Peking Union Medical College Hospital, Chinese Academy of Medical Sciences \& Peking Union Medical College) for his advice on data analysis data extraction. This work was supported by the National Natural Science Foundation of China (Grant No. 81970025).

Contributors ZZ led the meta-analysis was involved at every stage, including protocol development, screening, data extraction, quality assessment, data analysis and manuscript drafting. YL was involved in screening, data extraction, quality assessment, interpretation of results and manuscript revisions. JL facilitated manuscript revisions for important intellectual content. JG supervised this review and was involved in protocol preparation, consensus on disagreement in data extraction, quality assessment, data analysis, interpretation of results, manuscript drafting and revisions.

Funding This work was supported by the National Natural Science Foundation of China (81970025)

Competing interests None declared.

Patient consent for publication Not required.

Provenance and peer review Not commissioned; externally peer reviewed.

Data availability statement № data are available. No additional data are available.

Supplemental material This content has been supplied by the author(s). It has not been vetted by BMJ Publishing Group Limited (BMJ) and may not have been peer-reviewed. Any opinions or recommendations discussed are solely those of the author(s) and are not endorsed by BMJ. BMJ disclaims all liability and responsibility arising from any reliance placed on the content. Where the content includes any translated material, BMJ does not warrant the accuracy and reliability of the translations (including but not limited to local regulations, clinical guidelines, terminology, drug names and drug dosages), and is not responsible for any error and/or omissions arising from translation and adaptation or otherwise.

Open access This is an open access article distributed in accordance with the Creative Commons Attribution Non Commercial (CC BY-NC 4.0) license, which permits others to distribute, remix, adapt, build upon this work non-commercially, and license their derivative works on different terms, provided the original work is properly cited, appropriate credit is given, any changes made indicated, and the use is non-commercial. See: http://creativecommons.org/licenses/by-nc/4.0/.

ORCID iD

Jinming Gao http://orcid.org/0000-0003-1044-5284

\section{REFERENCES}

$1 \mathrm{Li} \mathrm{X}$, Cao X, Guo M, et al. Trends and risk factors of mortality and disability adjusted life years for chronic respiratory diseases from 
1990 to 2017: systematic analysis for the global burden of disease study 2017. BMJ 2020;368:m234.

2 Global Initiative for Asthma. Global strategy for asthma management and prevention, 2019. Available: www.ginasthma. org

3 Vakil N, van Zanten SV, Kahrilas P, et al. The Montreal definition and classification of gastroesophageal reflux disease: a global evidencebased consensus. Am J Gastroenterol 2006;101:1900-20.

4 Kiljander TO, Laitinen JO. The prevalence of gastroesophageal reflux disease in adult asthmatics. Chest 2004;126:1490-4.

5 Sandur V, Murugesh M, Banait V, et al. Prevalence of gastroesophageal reflux disease in patients with difficult to control asthma and effect of proton pump inhibitor therapy on asthma symptoms, reflux symptoms, pulmonary function and requirement for asthma medications. J Postgrad Med 2014;60:282-6.

6 Broers C, Tack J, Pauwels A. Review article: gastro-oesophageal reflux disease in asthma and chronic obstructive pulmonary disease. Aliment Pharmacol Ther 2018;47:176-91.

7 Harding SM. Gastroesophageal reflux and asthma: insight into the association论动呍 $\star$. J Allergy and Immunol 1999;104:251-9.

8 Zerbib F, Guisset $\mathrm{O}$, Lamouliatte $\mathrm{H}$, et al. Effects of bronchial obstruction on lower esophageal sphincter motility and gastroesophageal reflux in patients with asthma. Am J Respir Crit Care Med 2002;166:1206-11.

9 Gyawali CP, Fass R. Management of gastroesophageal reflux disease. Gastroenterology 2018;154:302-18.

10 Ford GA, Oliver PS, Prior JS, et al. Omeprazole in the treatment of asthmatics with nocturnal symptoms and gastro-oesophageal reflux: a placebo-controlled cross-over study. Postgrad Med J 1994;70:350-4.

11 Meier JH, McNally PR, Punja M. Does omeprazole (Prilosec) improve respiratory function in asthmatics with gastroesophageal reflux? A double-blind, placebo-controlled crossover study. Dig Dis Sci 1994;39:2127-33.

12 Teichtahl H, Kronborg IJ, Yeomans ND, et al. Adult asthma and gastro-oesophageal reflux: the effects of omeprazole therapy on asthma. Aust N Z J Med 1996;26:671-6.

13 Boeree MJ, Peters FTM, Postma DS, et al. No effects of high-dose omeprazole in patients with severe airway hyperresponsiveness and (a)symptomatic gastro-oesophageal reflux. Eur Respir J 1998;11:1070-4.

14 Levin TR, Sperling RM, McQuaid KR. Omeprazole improves peak expiratory flow rate and quality of life in asthmatics with gastroesophageal reflux. Am J Gastroenterol 1998;93:1060-3.

15 Kiljander TO, Salomaa ER, Hietanen EK. Gastroesophageal reflux in asthmatics: a double-blind, placebo-controlled crossover study with omeprazole. Chest 1999;116:1257-64.

16 Littner MR, Leung FW, Ballard ED, et al. Effects of 24 weeks of lansoprazole therapy on asthma symptoms, exacerbations, quality of life, and pulmonary function in adult asthmatic patients with acid reflux symptoms. Chest 2005;128:1128-35.

17 Størdal K, Johannesdottir GB, Bentsen BS, et al. Acid suppression does not change respiratory symptoms in children with asthma and gastro-oesophageal reflux disease. Arch Dis Child 2005;90:956-60.

18 Kiljander TO, Harding SM, Field SK, et al. Effects of esomeprazole 40 $\mathrm{Mg}$ twice daily on asthma: a randomized placebo-controlled trial. Am $J$ Respir Crit Care Med 2006;173:1091-7.

19 dos Santos LH, Ribeiro IOeS, Sánchez PG, et al. Evaluation of pantoprazol treatment response of patients with asthma and gastroesophageal reflux: a randomized prospective double-blind placebo-controlled study. J Bras Pneumol 2007;33:119-27.

20 Susanto $A D$, Yunus F, Wiyono WH, et al. Asthma symptoms improvement in moderate persistent asthma patients with gastroesophageal reflux disease (GERD): the role of proton-pump inhibitor. Medical Journal of Indonesia 2008;17:169-74.

21 American Lung Association Asthma Clinical Research Centers, Mastronarde JG, Anthonisen NR, et al. Efficacy of esomeprazole for treatment of poorly controlled asthma. N Engl J Med 2009;360:1487-99.

22 Kiljander TO, Junghard O, Beckman O, et al. Effect of esomeprazole $40 \mathrm{Mg}$ once or twice daily on asthma: a randomized, placebocontrolled study. Am J Respir Crit Care Med 2010;181:1042-8.

23 Writing Committee for the American Lung Association Asthma Clinical Research Centers, Holbrook JT, Wise RA, et al. Lansoprazole for children with poorly controlled asthma: a randomized controlled trial. JAMA 2012;307:373-80.

24 Coughlan JL, Gibson PG, Henry RL. Medical treatment for reflux oesophagitis does not consistently improve asthma control: a systematic review. Thorax 2001;56:198-204.

25 Gibson PG, Henry RL, Coughlan JL. Gastro-Oesophageal reflux treatment for asthma in adults and children. Cochrane Database Syst Rev 2003;2:Cd001496.

26 Sopo SM, Radzik D, Calvani M. Does treatment with proton pump inhibitors for gastroesophageal reflux disease (GERD) improve asthma symptoms in children with asthma and GERD? A systematic review. J Investig Allergol Clin Immunol 2009;19:1-5.

27 Chan WW, Chiou E, Obstein KL, et al. The efficacy of proton pump inhibitors for the treatment of asthma in adults. Arch Intern Med 2011;171:620-9.

28 Higgins JPT, Altman DG, Gotzsche PC, et al. The Cochrane Collaboration's tool for assessing risk of bias in randomised trials. BMJ 2011;343:d5928.

29 Santanello Nc, Zhang J, Seidenberg B, et al. What are minimal important changes for asthma measures in a clinical trial? Eur Respir J 1999;14:23-7.

30 Reddel HK, Taylor DR, Bateman ED. An official American thoracic Society/European respiratory Society statement: asthma control and exacerbations: standardizing endpoints for clinical asthma trials and clinical practice. Am J Respir Crit Care Med 2009;180:59-99.

31 Hom C, Vaezi MF. Extra-esophageal manifestations of gastroesophageal reflux disease: diagnosis and treatment. Drugs 2013;73:1281-95.

32 Naik RD, Vaezi MF. Extra-esophageal gastroesophageal reflux disease and asthma: understanding this interplay. Expert Rev Gastroenterol Hepatol 2015;9:969-82.

33 Vos R, Blondeau K, Vanaudenaerde BM, et al. Airway colonization and gastric aspiration after lung transplantation: do birds of a feather flock together? J Heart Lung Transplant 2008;27:843-9.

34 Griffin SM, Robertson AGN, Bredenoord AJ, et al. Aspiration and allograft injury secondary to gastroesophageal reflux occur in the immediate post-lung transplantation period (prospective clinical trial). Ann Surg 2013;258:705-12.

35 Peterson KA, Samuelson WM, Ryujin DT, et al. The role of gastroesophageal reflux in exercise-triggered asthma: a randomized controlled trial. Dig Dis Sci 2009;54:564-71.

36 Sharma B, Sharma M, Daga MK, et al. Effect of omeprazole and domperidone on adult asthmatics with gastroesophageal reflux. World J Gastroenterol 2007;13:1706-10.

37 Bediwy AS, Al-Biltagi M, Amer HG, et al. Combination therapy versus monotherapy for gastroesophageal reflux in children with difficult-totreat bronchial asthma. Egypt J Chest Dis Tuberc 2014;63:33-8.

38 Tang M, Blake KV, Lima JJ, et al. Genotype tailored treatment of mild symptomatic acid reflux in children with uncontrolled asthma (GenARA): rationale and methods. Contemp Clin Trials 2019;78:27-33. 